\title{
Average duration of prior treatment lines predicts clinical benefit to eribulin chemotherapy in patients with metastatic breast cancer
}

\author{
Faye Coe ${ }^{1} \cdot$ Vivek Misra $^{2} \cdot$ Yamini McCabe $^{3} \cdot$ Helen Adderley $^{3} \cdot$ Laura Woodhouse $^{3} \cdot$ Zaheen Ayub $^{1} \cdot$ Xin Wang $^{4} \cdot$ \\ Sacha Howell ${ }^{3} \cdot$ Maria Ekholm ${ }^{3,5,6}$ —
}

Received: 3 September 2021 / Accepted: 25 October 2021 / Published online: 29 November 2021

(c) The Author(s) 2021

\begin{abstract}
Purpose The aim of this study was to identify factors associated with progression-free survival (PFS) and overall survival (OS) in patients with metastatic breast cancer (MBC) treated with eribulin in a real-world setting, to improve information provision in those considering treatment.

Methods Patients treated with eribulin for MBC at The Christie NHS Foundation Trust, Manchester, UK, between August 2011 and December 2018 were included $(n=439)$. Data were collected by retrospective review of medical records and electronic prescribing systems. Factors such as biological subtype, distant recurrence-free interval, previous lines of chemotherapy and the 'average duration of previous treatment lines' (ADPT) (calculated as: (date of initiation of eribulin-date of $\mathrm{MBC}$ ) / the number of previous treatment lines in the metastatic setting) were evaluated for prognostic impact using Cox proportional hazards regression.

Results In the full cohort, the median PFS and OS were 4.1 months (95\% CI 3.7-4.4) and 8.6 months (95\% CI 7.4-9.8), respectively. Outcomes were significantly inferior for those with triple-negative breast cancer (TNBC) $(n=92)$; PFS $_{\text {TNBC: }}$ : 2.4 months (95\% CI 2.1-3.0), $p=<0.001$ and $\mathrm{OS}_{\mathrm{TNBC}}$ : 5.4 months (95\% CI 4.6-6.6), $p=<0.001$. ADPT was the only factor other than subtype significantly associated with PFS and OS. Longer ADPT was also significantly associated with PFS and OS in those with TNBC. For example, women in the lowest ADPT tertile $(<5.0$ months $)$ achieved a median OS of only 4.3 months, whereas those in the upper ADPT tertile ( $>8.7$ months) had a median OS of 12.1 months ( $p=0.004)$.

Conclusion Our results indicate that the ADPT lines is an important factor when predicting the outcome with eribulin chemotherapy in a palliative setting and that quantitative guidance on the likely PFS and OS with treatment can be provided using ADPT. Validation in additional cohorts is warranted.
\end{abstract}

Keywords Metastatic breast cancer $\cdot$ Eribulin $\cdot$ Real world $\cdot$ Subtypes $\cdot$ Chemotherapy $\cdot$ Palliative

Maria Ekholm

maria.ekholm@rjl.se

1 Department of Pharmacy, The Christie NHS Foundation Trust, Manchester, UK

2 Department of Clinical Oncology, The Christie NHS Foundation Trust, Manchester, UK

3 Department of Medical Oncology, The Christie NHS Foundation Trust, Manchester, UK

4 Department of Analytics and Statistics, Digital Services, The Christie NHS Foundation Trust, Manchester, UK

5 Department of Oncology, Ryhov Hospital, Jönköping, Sweden

6 Institute of Biomedicine, Department of Laboratory Medicine, Sahlgrenska Center for Cancer Research, Sahlgrenska Academy at University of Gothenburg, Gothenburg, Sweden

\section{Introduction}

Breast cancer is the most common cancer in women with approximately 1.7 million new cases per year worldwide [1]. The aim of systemic therapy for metastatic breast cancer (MBC) is to delay disease progression, improve overall survival (OS) and at the same time maintain or improve the quality of life by controlling cancer-related symptoms. Although clear guidelines exist for first-line treatment options, later treatment lines are less well evidenced, potentially less efficacious and therefore the side effect profile and patient wishes are highly important to take into consideration when choosing treatment [2].

Eribulin, a non-taxane microtubule dynamics inhibitor, was approved for the treatment of advanced breast cancer 
based on two phase III studies [3, 4]. In EMBRACE, heavily pretreated patients with $\mathrm{MBC}$ of all subtypes were randomised 2:1 to receive eribulin or treatment of physician's choice (TPC) [3]. Progression-free survival (PFS) was 3.7 months in the eribulin arm and eribulin was shown to improve OS significantly compared to TPC (13.1 months vs. 10.6 months; Hazard Ratio (HR) $=0.81, p=0.041$ ) [3] In Study 301, patients with MBC of all subtypes previously treated with anthracyclines and taxanes were assigned 1:1 to receive eribulin or capecitabine as 1st-, 2nd- or 3rd-line therapy. In this trial, PFS was 4.1 months and no significant difference in OS was seen between the 2 groups (15.9 months vs. 14.5 months, $p=0.056$ ) [4]. However, a subgroup analysis of patients with human epidermal growth factor receptor 2 (HER2) negative MBC did show a longer OS in the eribulin arm (16.1 months vs. 13.5 months, $p=0.026$ ) [5]. A pooled analysis of EMBRACE and Study 301 has also been published ( $n=1864$; eribulin $n=1062$, TPC or capecitabine $n=802)$ with OS data favouring eribulin (15.2 months vs. 12.8 months, $p=0.003$ ) and a similar positive effect seen across the different subtypes [6].

The primary aim of the current study was to identify factors associated with PFS and OS in patients with different subtypes of MBC treated with eribulin in a real-world setting to improve information provision to patients considering palliative treatment in the metastatic setting.

\section{Methods}

\section{Patients}

All patients treated with eribulin for MBC at The Christie NHS Foundation Trust (The Christie), Manchester, UK, between 1st August 2011 and 31st December 2018 were identified through the electronic patient data systems. Medical records and electronic prescribing systems were reviewed by four doctors (ME, YM, LW and HA) and two pharmacists (FC and ZA). Data on breast cancer history and previous treatments, patient and tumour characteristics, eribulin treatment, hospital admissions and outcome were collected using a predefined case report form. Last date for follow-up was 30th of March 2020. Detailed information on adverse events was not collected. The majority of patients were treated with the standard dose of eribulin, i.e. $1.23 \mathrm{mg} / \mathrm{m}^{2}$ on days 1 and 8 in a 21 -day cycle; however, but those who recieved dose reductions from cycle 1 are included. Radiological assessment was performed as per standard of care, generally every 4 cycles. Patients were divided into the following subtypes based on the biological characteristics of the tumour; oestrogen receptor (ER) positive and/or progesterone receptor (PR) positive and HER2 negative (ER + /HER2-); HER2 positive, irrespective of ER/PR status (HER2+); ER-/PR-/HER2- (TNBC). ER/PR positivity was defined as $\geq 1 \%$ positively stained nuclei or quick score $(\mathrm{QS}) \geq 3$ if no percentage had been recorded.

\section{Statistical analyses}

Summary statistics were provided for patient and tumour characteristics. Median Tests were applied for continuous variables and Chi-squared Tests were applied for categorical variables to assess the differences between subtype groups for corresponding variables. PFS was defined as the time from start of eribulin treatment until progressive disease, primarily radiological but clinical for patients who clearly had progressive disease without undergoing a scan, or death from any cause. OS was defined as the time from start of treatment until death from any cause. Patients lost to followup were censored at the day of their last eribulin treatment for PFS and the day of their last follow-up for OS. Distant recurrence-free interval (DRFi) was defined as the date of the primary cancer until diagnosis of distant recurrence. The 'average duration of previous treatment lines' (ADPT) was calculated as: (date of initiation of eribulin-date of $\mathrm{MBC}$ ) / the number of previous treatment lines in the metastatic setting, including endocrine therapy for patients with $\mathrm{ER}+$ disease. Treatments given $<1$ month, irrespectively of stop cause, was disregarded when counting the number of previous treatment lines. The Kaplan-Meier method was used to estimate median survival and corresponding $95 \%$ confidence intervals (CIs). Univariable Cox proportional hazards $(\mathrm{PH})$ regression was applied to assess the association between PFS and OS, respectively, and DRFi, number of previous chemotherapy lines for MBC, and ADPT categorised as tertiles for the whole group and subgroups separately and as continuous variables. Patients with de novo metastatic disease were treated as 'no record' in the calculation of DRFi and they were excluded when calculating the tertiles for DRFi. Patients who received eribulin as first line (because of recurrence shortly after having completed adjuvant therapy, including an anthracycline and a taxane) were treated as 'no record' in the calculation of ADPT and they were excluded from the calculation of the tertiles for ADPT. The association between PFS and OS and cancer subtypes were assessed using Cox PH regression. Hazard ratios together with their corresponding $95 \%$ confidence intervals and Wald $P$ values were calculated. All presented $P$ values are two-sided. Statistical analyses were performed using R version 3.6.2 (2019 The R Foundation for Statistical Computing). 


\section{Results}

In total, 439 patients commenced eribulin for $\mathrm{MBC}$ at The Christie between 1st August 2011 and 31st December 2018. Patient characteristics and breast cancer history for all patients and divided by subtype are summarised in
Table 1. In total, $44.0 \%(n=193 / 439)$ of the patients had a dose reduction of eribulin, $12.5 \%(n=55 / 439)$ were given granulocyte colony-stimulating factor (G-CSF) and $8.2 \%(n=36 / 439)$ had both a dose reduction and G-CSF. In patients with HER $2+\mathrm{MBC}, 7.4 \%$ (5/68) received trastuzumab concomitantly with eribulin. During the treatment period $48.3 \%(n=212 / 439)$ patients were admitted

Table 1 Patient and tumour characteristics at start of eribulin therapy, for all patients and divided by biological subtype

\begin{tabular}{|c|c|c|c|c|c|}
\hline & All patients & ER +/HER2- & HER2 + & TNBC & $P$-value \\
\hline Number of patients (\%) & $439(100.0)$ & $279(63.6)$ & $68(15.5)$ & $92(21.0)$ & \\
\hline \multicolumn{6}{|l|}{ DRFi, years } \\
\hline Median (min-max) & $53.0(2.0-497.5)$ & $75.9(2.0-497.5)$ & $37.9(4.0-352.3)$ & $23.0(4.0-282.2)$ & \multirow[t]{5}{*}{$<0.001^{\mathrm{a}}$} \\
\hline 1st tertile & $\leq 2.8$ & $\leq 4.3$ & $\leq 2.4$ & $\leq 1.4$ & \\
\hline 2nd tertile & $>2.8-7.0$ & $>4.3-8.8$ & $>2.4-4.9$ & $>1.4-2.8$ & \\
\hline 3rd tertile & $>7.0$ & $>8.8$ & $>4.9$ & $>2.8$ & \\
\hline De novo metastatic & $75(17.1)$ & $47(16.8)$ & $14(20.6)$ & $13(14.1)$ & \\
\hline \multicolumn{6}{|l|}{ Age } \\
\hline Median (range) & $56(32-87)$ & $56(34-87)$ & $56(40-81)$ & $54(32-81)$ & $0.11^{\mathrm{a}}$ \\
\hline \multicolumn{6}{|l|}{ ECOG, n (\%) } \\
\hline 0 & $137(48.8)$ & $88(48.4)$ & $21(52.5)$ & $28(47.5)$ & \multirow[t]{5}{*}{$0.98^{\mathrm{b}}$} \\
\hline 1 & $104(37.0)$ & $69(37.9)$ & $13(32.3)$ & $22(37.3)$ & \\
\hline 2 & $37(13.2)$ & $23(12.6)$ & $6(15.0)$ & $8(13.6)$ & \\
\hline 3 & $3(0.1)$ & $2(0.1)$ & $0(0)$ & $1(0.2)$ & \\
\hline Missing & 158 & 97 & 28 & 33 & \\
\hline \multicolumn{6}{|l|}{ Metastatic sites } \\
\hline Median, $\mathrm{n}$ & 3 & 3 & 3 & 2 & $0.12^{\mathrm{a}}$ \\
\hline \multicolumn{6}{|l|}{ Sites of metastases } \\
\hline Bone & $302(68.8)$ & $223(79.9)$ & $42(61.8)$ & $37(40.2)$ & $<0.001^{\mathrm{b}}$ \\
\hline Lung/pleura & $245(55.8)$ & $150(53.8)$ & $38(55.9)$ & $57(62.0)$ & $0.39^{\mathrm{b}}$ \\
\hline Liver & $268(61.1)$ & $200(71.7)$ & $32(47.1)$ & $36(39.1)$ & $<0.001^{\mathrm{b}}$ \\
\hline Lymph nodes & $198(45.1)$ & $109(39.1)$ & $39(57.4)$ & $50(54.4)$ & $0.003^{\mathrm{b}}$ \\
\hline CNS & $77(17.5)$ & $28(13.6)$ & $22(32.4)$ & $17(18.5)$ & $0.001^{\mathrm{b}}$ \\
\hline Other & $145(33.0)$ & $76(27.2)$ & $30(44.1)$ & $39(42.4)$ & $0.003^{\mathrm{b}}$ \\
\hline \multicolumn{6}{|c|}{$\begin{array}{l}\text { Previous number of treatment } \\
\text { lines for metastatic disease }\end{array}$} \\
\hline Median (range) & $3(0-11)$ & $4(0-11)$ & $3(1-11)$ & $2(0-5)$ & $<0.001^{\mathrm{a}}$ \\
\hline \multicolumn{6}{|c|}{$\begin{array}{l}\text { Previous number of chemo- } \\
\text { therapy regimens for meta- } \\
\text { static disease }\end{array}$} \\
\hline Median (range) & $2(0-8)$ & $2(0-8)$ & $3(1-7)$ & $2(0-5)$ & $0.004^{\mathrm{a}}$ \\
\hline$\leq 2$ & $274(62.4)$ & $175(62.7)$ & $32(54.4)$ & $67(72.8)$ & $0.004^{\mathrm{b}}$ \\
\hline$>2$ & 165 (37.6) & $104(37.3)$ & $36(52.9)$ & $25(27.2)$ & \\
\hline \multicolumn{6}{|l|}{ ADPT, months } \\
\hline Median (min-max) & $8.0(1.6-48.4)$ & $9.6(3.2-27.8)$ & $9.6(3.2-28.8)$ & $6.7(0.3-31.4)$ & $0.031^{\mathrm{a}}$ \\
\hline 1st tertile & $\leq 6.3$ & $\leq 6.5$ & $\leq 7.8$ & $\leq 5.0$ & \\
\hline 2nd tertile & $>6.3-10.4$ & $>6.5-10.43$ & $>7.8-12.4$ & $>5.0-8.7$ & \\
\hline 3rd tertile & $>10.4$ & $>10.4$ & $>12.4$ & $>8.7$ & \\
\hline $\mathrm{NA}^{4}$ & 2 & 2 & 0 & 1 & \\
\hline
\end{tabular}

$A D P T$ average duration of previous treatment lines, $D R F i$ distant recurrence-free interval, $N A$ not applicable

${ }^{\text {a Median Test }}$

${ }^{\mathrm{b}}$ Chi-squared Test 
to hospital at least one time. Reasons for hospital admissions are listed in Table 2 along with reasons for eribulin discontinuation. The median OS from the date of MBC diagnosis (MBC OS) was 41.3 months (95\% CI 38.1-44.3) for all patients and differed between the biological subtypes; $\mathrm{MBC} \mathrm{OS}_{\mathrm{ER}+\text { /HER2 }-}: 46.7$ months (95\% CI 43.3-51.7), $\mathrm{MBC} \mathrm{OS}_{\mathrm{HER} 2+}: 48.5$ months (95\% CI 38.0-60.1) and MBC $\mathrm{OS}_{\mathrm{TNBC}}: 22.0$ months (95\% CI 18.7-25.7), $p=<0.001$. These results must be interpreted with caution as they are affected by survivor bias, since only those patients who survived to receive at least one dose of eribulin were included in the cohort.

\section{Survival analyses}

The median PFS and OS with eribulin for the whole cohort were 4.1 months (95\% CI 3.7-4.4) and 8.6 months (95\% CI 7.4-9.8), respectively. Outcomes were significantly inferior for those with TNBC; $\mathrm{PFS}_{\mathrm{ER}+/ \mathrm{HER} 2-}$ : 4.6 months $(95 \%$

Table 2 Information on treatment and adverse events

\begin{tabular}{ll}
\hline & $n(\%)$ \\
\hline Number of patients (\%) & $439(100)$ \\
Dose reduction & \\
No & $246(56.0)$ \\
Yes, once & $71(16.2)$ \\
Yes, multiple & $122(27.8)$ \\
G-CSF & \\
No & $384(87.5)$ \\
Yes, at C1 & $19(4.3)$ \\
Yes, after C1 & $36(8.2)$ \\
Hospital admission ${ }^{\mathrm{a}}$ & \\
Yes & $212(48.3)$ \\
Reason(s) for hospital admission & \\
Febrile neutropenia & $54(12.3)$ \\
Non-neutropenicinfection & $85(19.4)$ \\
Eribulin-related toxicity & $48(10.9)$ \\
Other reason & $106(24.2)$ \\
Treatment-related death & \\
Yes & $3(0.7)$ \\
Reason for discontinuation & \\
Disease progression & $340(78.7)$ \\
Toxicity & $24(5.6)$ \\
Not fit for further treatment & $34(7.9)$ \\
Physician's or patient's choice & $9(2.1)$ \\
Other & $1(0.2)$ \\
Death & $24(5.6)$ \\
Lost to follow-up & 4 \\
NA (ongoing treatment at data cut-off) & 3 \\
\hline
\end{tabular}

${ }^{a}$ Number of patients admitted, not total number of admissions

${ }^{b}$ Patients may have had several reasons for admission
CI 4.2-5.2); $\mathrm{PFS}_{\mathrm{HER} 2+}: 3.9$ months $(95 \%$ CI 2.9-5.5) and PFS $_{\text {TNBC }}: 2.4$ months (95\% CI 2.1-3.0), $p<0.001$ (Table 3) and $\mathrm{OS}_{\mathrm{ER}+/ \mathrm{HER} 2-:} 9.5$ months (95\% CI 8.3-11.1); $\mathrm{OS}_{\mathrm{HER} 2+}$ : 9.2 months (95\% CI 6.9-13.0) and $\mathrm{OS}_{\mathrm{TNBC}}: 5.4$ months (95\% CI 4.6-6.6), $p<0.001$ ) (Table 4).

\section{Prognostic factors and eribulin efficacy}

Factors that were explored for eribulin efficacy are presented in Tables 3 and 4 for PFS and OS, respectively. Longer ADPT was positively associated with PFS for all patients; $\mathrm{ADPT}_{1 \mathrm{st} \text { tertile }}\left(\leq 6.3\right.$ months): ref, $\mathrm{ADPT}_{2 \text { nd tertile }}$ ( $>6.3-10.1$ months): $\mathrm{HR}=0.72, p=0.009, \mathrm{ADPT}_{3 \mathrm{rd}}$ tertile ( $>10.1$ months): $\mathrm{HR}=0.62, p=<0.001$ and $\mathrm{ADPT}_{\text {linear }}$ : $\mathrm{HR}=0.96, p=<0.001$. Similar results were seen for OS; $\mathrm{ADPT}_{1 \mathrm{st} \text { tertile }}$ ref, $\mathrm{ADPT}_{2 \text { nd tertile }}: \mathrm{HR}=0.80, p=0.061$, $\mathrm{ADPT}_{3 \text { rd tertile: }}: \mathrm{HR}=0.64, p=<0.001$ and ADPT linear: $\mathrm{HR}=0.96, \mathrm{p}=<0.001$. Longer ADPT was also positively associated with PFS and OS in the different biological subtypes, reaching statistical significance in the TNBC subgroup (Tables 3 and 4).

\section{Discussion}

The current study represents the largest single-institution experience of the use of eribulin in MBC and includes data on outcomes for the different biological subtypes. For the whole cohort, PFS was similar but OS was somewhat shorter than in the previously published randomised trials [3, 4]. Our cohort was more heavily pretreated when compared to Study 301 [4] and 41 patients (9.3\%) received $\leq 1$ full cycle of eribulin and had PFS and OS of only 0.6 months (95\% CI 0.5-1.2) and 1.4 months (95\% CI 1.1-2.2), respectively. These patients may have been better suited for best supportive care and are unlikely to have fulfilled the eligibility criteria for clinical trials. The results of the prognostic analyses in the study were largely unchanged after exclusion of these individuals (data not shown). Other trials based on real-world data including $>100$ patients report a wider spread of results for PFS (3.3-6.1 months) and OS (10.6-31.8 months) [7-14]. Differences in PFS could result from less frequent imaging or patient and tumour variability as well as differences in previous lines of MBC treatment between the cohorts. For example, Adamo et al. reported PFS of 5.5 months and OS of 31.8 months, which may be the result of $70 \%$ patients in their cohort having cancers that the authors classified as luminal A subtype [13].

As expected, the median PFS and OS in our cohort differed between the biological subtypes and were inferior in those with TNBC (Tables 3 and 4). Importantly, the outcomes for patients with HER 2 + disease may not be comparable to present day worldwide expectations for two 
Table 3 Median progression-free survival and univariable analysis of prognostic factors for progression-free survival for all patients and divided by biological subtype

\begin{tabular}{|c|c|c|c|c|c|}
\hline & $\begin{array}{l}\text { Number of } \\
\text { events/patients }\end{array}$ & $\begin{array}{l}\text { Median PFS, months } \\
(95 \% \text { CI })\end{array}$ & Log rank $p$-value & Hazard Ratio (95\% CI) & Wald $p$-value \\
\hline All patients & $364 / 439$ & $4.1(3.7-4.4)$ & & & \\
\hline $\mathrm{ER}+/ \mathrm{HER} 2-$ & $229 / 279$ & $4.6(4.2-5.2)$ & $<0.001$ & Ref. & \\
\hline HER2 & $57 / 68$ & $3.9(2.9-5.5)$ & & $1.03(0.77-1.38)$ & 0.84 \\
\hline TNBC & $78 / 92$ & $2.4(2.1-3.0)$ & & $1.98(1.53-2.57)$ & $<0.001$ \\
\hline \multicolumn{6}{|l|}{ All patients } \\
\hline \multicolumn{6}{|l|}{ DRFi, years } \\
\hline 1st tertile $(\leq 2.8)$ & $99 / 122$ & $2.8(2.3-4.4)$ & 0.30 & Ref. & \\
\hline 2nd tertile $(>2.8-7.0)$ & $102 / 121$ & $4.4(3.7-5.0)$ & & $0.90(0.68-1.19)$ & 0.47 \\
\hline 3rd tertile $(>7.0)$ & $102 / 121$ & $3.9(3.8-5.3)$ & & $0.79(0.60-1.05)$ & 0.10 \\
\hline De novo metastatic & $61 / 75$ & $3.9(3.4-4.6)$ & & $1.03(0.74-1.42)$ & 0.87 \\
\hline Linear & & & & $0.99(0.97-1.01)$ & 0.21 \\
\hline \multicolumn{6}{|c|}{ Previous chemotherapy lines, $\mathrm{n}$} \\
\hline$\leq 2$ & $227 / 274$ & $4.3(3.7-4.6)$ & 0.65 & Ref. & \\
\hline$>2$ & $137 / 165$ & $3.9(3.6-4.6)$ & & $1.05(0.85-1.07)$ & 0.66 \\
\hline Linear & & & & $0.94(0.86-1.03)$ & 0.19 \\
\hline \multicolumn{6}{|l|}{ ADPT, months } \\
\hline 1st tertile $(\leq 6.3)$ & $126 / 146$ & $2.8(2.3-3.9)$ & 0.001 & Ref. & \\
\hline 2nd tertile $(>6.3-10.1)$ & $124 / 145$ & $4.4(3.8-5.0)$ & & $0.72(0.56-0.92)$ & 0.009 \\
\hline 3rd tertile $(>10.1)$ & $111 / 145$ & $4.7(4.0-5.3)$ & & $0.62(0.42-0.69)$ & $<0.001$ \\
\hline Linear & & & & $0.96(0.94-0.98)$ & $<0.001$ \\
\hline \multicolumn{6}{|l|}{$\mathrm{ER}+/ \mathrm{HER} 2-$} \\
\hline \multicolumn{6}{|l|}{ DRFi, years } \\
\hline 1st tertile $(\leq 4.3)$ & $63 / 78$ & $4.4(3.7-7.1)$ & 0.44 & Ref. & \\
\hline 2nd tertile $(>4.3-8.8)$ & $65 / 77$ & $5.0(4.4-6.1)$ & & $1.06(0.75-1.51)$ & 0.73 \\
\hline 3rd tertile $(>8.8)$ & $67 / 77$ & $4.6(3.6-5.3)$ & & $1.29(0.91-1.82)$ & 0.16 \\
\hline De novo metastatic & $34 / 47$ & $4.4(3.7-7.3)$ & & $1.27(0.83-1.94)$ & 0.27 \\
\hline Linear & & & & $1.01(0.99-1.03)$ & 0.45 \\
\hline \multicolumn{6}{|c|}{ Previous chemotherapy lines, $\mathrm{n}$} \\
\hline$\leq 2$ & $143 / 175$ & $4.6(3.7-5.3)$ & 0.91 & Ref. & \\
\hline$>2$ & $86 / 104$ & $4.6(4.3-5.3)$ & & $0.99(0.75-1.29)$ & 0.91 \\
\hline Linear & & & & $0.91(0.80-1.02)$ & 0.11 \\
\hline \multicolumn{6}{|l|}{ ADPT, months } \\
\hline 1 st tertile $(\leq 6.5)$ & $79 / 93$ & $4.1(3.0-5.2)$ & 0.36 & Ref. & \\
\hline 2nd tertile $(>6.5-10.4)$ & $80 / 92$ & $5.3(4.4-7.2)$ & & $0.80(0.58-1.09)$ & 0.15 \\
\hline 3rd tertile $(>10.4)$ & $68 / 92$ & $4.6(3.7-5.3)$ & & $0.88(0.64-1.22)$ & 0.45 \\
\hline Linear & & & & $0.98(0.95-1.00)$ & 0.039 \\
\hline \multicolumn{6}{|l|}{$\mathrm{HER} 2+$} \\
\hline \multicolumn{6}{|l|}{ DRFi, years } \\
\hline 1st tertile $(\leq 2.4)$ & $14 / 18$ & $5.0(2.5-*)$ & 0.62 & Ref. & \\
\hline 2nd tertile $(>2.4-4.9)$ & $14 / 17$ & $3.7(2.1-7.5)$ & & $1.65(0.75-3.6)$ & 0.21 \\
\hline 3rd tertile $(>4.9)$ & $14 / 18$ & $3.1(1.8-*)$ & & $1.24(0.58-2.66)$ & 0.58 \\
\hline De novo metastatic & $15 / 15$ & $3.9(3.4-9.1)$ & & $1.46(0.68-3.13)$ & 0.33 \\
\hline Linear & & & & $0.97(0.92-1.03)$ & 0.35 \\
\hline \multicolumn{6}{|c|}{ Previous chemotherapy lines, $\mathrm{n}$} \\
\hline$\leq 2$ & $27 / 32$ & $5.0(2.3-7.5)$ & 0.21 & Ref. & \\
\hline$>2$ & $30 / 36$ & $3.6(2.5-5.5)$ & & $1.40(0.82-2.38)$ & 0.22 \\
\hline Linear & & & & $1.06(0.87-1.28)$ & 0.57 \\
\hline
\end{tabular}


Table 3 (continued)

\begin{tabular}{|c|c|c|c|c|c|}
\hline & $\begin{array}{l}\text { Number of } \\
\text { events/patients }\end{array}$ & $\begin{array}{l}\text { Median PFS, months } \\
(95 \% \text { CI })\end{array}$ & Log rank $p$-value & Hazard Ratio (95\% CI) & Wald $p$-value \\
\hline \multicolumn{6}{|l|}{ ADPT, months } \\
\hline 1st tertile $(\leq 7.8)$ & $18 / 23$ & $2.9(1.8-6.7)$ & 0.19 & Ref. & \\
\hline 2nd tertile $(>7.8-12.4)$ & $20 / 22$ & $2.3(2.1-6.9)$ & & $0.71(0.37-1.36)$ & 0.30 \\
\hline 3rd tertile $(>12.4)$ & $19 / 23$ & $5.0(4.1-9.1)$ & & $0.55(0.29-1.05)$ & 0.071 \\
\hline Linear & & & & $0.95(0.90-1.00)$ & 0.063 \\
\hline \multicolumn{6}{|l|}{ TNBC } \\
\hline \multicolumn{6}{|l|}{ DRFi, years } \\
\hline 1st tertile $(\leq 1.4)$ & $23 / 27$ & $2.3(1.8-2.6)$ & 0.004 & Ref. & \\
\hline 2nd tertile $(>1.4-2.8)$ & $21 / 26$ & $2.3(1.7-4.5)$ & & $0.49(0.26-0.92)$ & 0.026 \\
\hline 3rd tertile $(>2.8)$ & $22 / 26$ & $3.8(3.0-6.0)$ & & $0.32(0.17-0.60)$ & $<0.001$ \\
\hline De novo metastatic & $12 / 13$ & $2.2(1.9-*)$ & & $0.68(0.34-1.39)$ & 0.29 \\
\hline Linear & & & & $0.93(0.85-1.01)$ & 0.075 \\
\hline \multicolumn{6}{|c|}{ Previous chemotherapy lines, $\mathrm{n}$} \\
\hline$\leq 2$ & $57 / 67$ & $2.4(2.1-3.0)$ & 0.48 & Ref. & \\
\hline$>2$ & $21 / 25$ & $2.6(1.9-3.9)$ & & $1.20(0.72-1.99)$ & 0.49 \\
\hline Linear & & & & $0.99(0.76-1.28)$ & 0.92 \\
\hline \multicolumn{6}{|l|}{ ADPT, months } \\
\hline 1st tertile $(\leq 5.0)$ & $28 / 31$ & $1.9(1.5-2.7)$ & 0.001 & Ref. & \\
\hline 2nd tertile $(>5.0-8.7)$ & $24 / 31$ & $2.2(1.9-4.4)$ & & $0.60(0.35-1.05)$ & 0.074 \\
\hline 3rd tertile $(>8.7)$ & $25 / 30$ & $3.8(2.6-5.0)$ & & $0.34(0.19-0.62)$ & $<0.001$ \\
\hline Linear & & & & $0.92(0.86-0.97)$ & 0.002 \\
\hline
\end{tabular}

$A D P T$ average duration of previous treatment lines, $D R F i$ distant recurrence-free interval, $P F S$ progression-free survival

reasons. Firstly, a significant proportion of these patients did not receive HER2 dual-blockade as $1^{\text {st }}$-line metastatic treatment, which is known to significantly increase OS [15]. Secondly, at the time of this project the National Institute for Health and Social Care Excellence (NICE) stipulated that only two lines of anti-HER2 therapy could be used in the metastatic setting. Therefore, most patients with HER2 + cancers only had two lines of anti-HER2 therapy unless additional lines were received in the context of clinical trials or private care. The vast majority did not have anti-HER 2 treatment concomitant with eribulin, which remains the treatment paradigm within the National Health Service (NHS) in England.

Based on the pooled data from EMBRACE and Study 301 reported by Cortes et al., the median OS was longer for patients having $\leq 3$ previous chemotherapy lines [16]. This may be expected as OS is generally longer for patients who are early into their disease. However, we did not demonstrate significant differences in OS between patients having $\leq 2$ vs. $>2$ previous chemotherapy lines for $\mathrm{MBC}$, nor for $\leq 3$ vs. $>3$ previous chemotherapy lines (data not shown for the latter). Instead, we hypothesised that it was important to not only assess the number of previous treatment lines but also to take into consideration their duration as a surrogate for efficacy. We therefore defined the variable ADPT. We found longer ADPT to be associated with better outcomes in all patients, both with regards to PFS and OS. Similar results were demonstrated for all biological subgroups, but the strongest correlation was found in TNBC. This is perhaps the case as prior lines in those with TNBC will have been chemotherapy regimens whereas in those with ER + /HER2- and HER2 + cancers, endocrine therapies and HER2-targeted agents will have contributed significantly with potentially non-overlapping mechanisms of resistance.

This study has several strengths. It is the largest singleinstitution real-world eribulin study, also reporting on outcomes for each biological subtype. The data were collected by review of medical records rather than register data and to our knowledge; we are the first to report on the importance of the duration of previous treatments. Although ADPT is not an established endpoint, our results indicate that the duration of prior therapy is important when predicting the benefit of chemotherapy in a palliative setting, rather than the number of previous chemotherapy lines per se. For patients living with MBC it may be very useful to know what to expect from a particular choice of therapy. For example, according to our data, a patient with TNBC and an ADPT of 3 months can only expect a PFS on average of less 
Table 4 Median overall survival and univariable analysis of prognostic factors for overall survival for all patients and divided by biological subtype

\begin{tabular}{|c|c|c|c|c|c|}
\hline & $\begin{array}{l}\text { Number of } \\
\text { events/patients }\end{array}$ & $\begin{array}{l}\text { Median OS, months } \\
(95 \% \mathrm{CI})\end{array}$ & Log rank $p$-value & Hazard Ratio (95\% CI) & Wald $p$-value \\
\hline All patients & $408 / 439$ & $8.6(7.4-9.8)$ & & & \\
\hline $\mathrm{ER}+/ \mathrm{HER} 2-$ & $255 / 279$ & $9.5(8.3-11.1)$ & $<0.001$ & Ref. & \\
\hline $\mathrm{HER}+$ & $64 / 68$ & $9.2(6.9-13.0)$ & & $1.08(0.82-1.42)$ & 0.60 \\
\hline TNBC & $89 / 92$ & $5.4(4.6-6.6)$ & & $1.66(1.30-2.13)$ & $<0.001$ \\
\hline \multicolumn{6}{|l|}{ All patients } \\
\hline \multicolumn{6}{|l|}{ DRFi, years } \\
\hline 1st tertile $(\leq 4.3)$ & $113 / 122$ & $6.4(5.8-9.2)$ & 0.20 & Ref. & \\
\hline 2nd tertile $(>4.3-8.8)$ & $115 / 121$ & $10.0(8.5-13.5)$ & & $0.84(0.64-1.09)$ & 0.18 \\
\hline 3rd tertile $(>8.8)$ & $108 / 121$ & $9.4(7.3-12.6)$ & & $0.78(0.59-1.01)$ & 0.060 \\
\hline De novo metastatic & $72 / 75$ & $8.2(6.1-10.4)$ & & $0.98(0.73-1.32)$ & 0.88 \\
\hline Linear & & & & $0.99(0.97-1.01)$ & 0.21 \\
\hline \multicolumn{6}{|c|}{ Previous chemotherapy lines, $n$} \\
\hline$\leq 2$ & $250 / 274$ & $8.8(7.3-10.7)$ & 0.065 & Ref. & \\
\hline$>2$ & $158 / 165$ & $8.2(6.4-9.7)$ & & $1.21(0.99-1.48)$ & 0.065 \\
\hline Linear & & & & $1.02(0.94-1.11)$ & 0.64 \\
\hline \multicolumn{6}{|l|}{ ADPT, months } \\
\hline 1st tertile $(\leq 6.5)$ & $138 / 146$ & $6.3(5.4-7.3)$ & 0.002 & Ref. & \\
\hline 2nd tertile $(>6.5-10.4)$ & $137 / 145$ & $9.2(7.7-10.9)$ & & $0.80(0.63-1.01)$ & 0.061 \\
\hline 3rd tertile $(>10.4)$ & $131 / 145$ & $11.6(9.4-14.5)$ & & $0.64(0.51-0.82)$ & $<0.001$ \\
\hline Linear & & & & $0.96(0.94-0.98)$ & $<0.001$ \\
\hline \multicolumn{6}{|l|}{ ER +/HER- } \\
\hline \multicolumn{6}{|l|}{ DRFi, years } \\
\hline 1st tertile $(\leq 4.3)$ & $68 / 78$ & $10.2(8.3-11.6)$ & 0.44 & Ref. & \\
\hline 2nd tertile $(>4.3-8.8)$ & $73 / 77$ & $10.4(7.3-15.3)$ & & $0.96(0.69-1.34)$ & 0.83 \\
\hline 3rd tertile $(>8.8)$ & $70 / 77$ & $9.5(7.1-13.4)$ & & $1.14(0.81-1.60)$ & 0.45 \\
\hline De novo metastatic & $44 / 47$ & $7.9(5.9-13.9)$ & & $1.28(0.87-1.87)$ & 0.21 \\
\hline Linear & & & & $1.00(0.98-1.02)$ & 0.80 \\
\hline \multicolumn{6}{|c|}{ Previous chemotherapy lines, $n$} \\
\hline$\leq 2$ & $156 / 175$ & $10.2(8.2-12.3)$ & 0.14 & Ref. & \\
\hline$>2$ & $99 / 104$ & $8.7(7.0-11.4)$ & & $1.21(0.94-1.56)$ & 0.14 \\
\hline Linear & & & & $1.02(0.92-1.13)$ & 0.70 \\
\hline \multicolumn{6}{|l|}{ ADPT, months } \\
\hline 1st tertile $(\leq 6.5)$ & $87 / 93$ & $7.9(6.8-10.6)$ & 0.14 & Ref. & \\
\hline 2nd tertile $(>6.5-10.4)$ & $85 / 92$ & $10.6(8.5-13.5)$ & & $0.81(0.60-1.10)$ & 0.17 \\
\hline 3rd tertile $(>10.4)$ & $81 / 92$ & $10.7(7.5-14.8)$ & & $0.76(0.56-1.04)$ & 0.083 \\
\hline Linear & & & & $0.98(0.95-1.00)$ & 0.042 \\
\hline \multicolumn{6}{|l|}{ HER2 + } \\
\hline \multicolumn{6}{|l|}{ DRFi, years } \\
\hline 1st tertile $(\leq 2.4)$ & $15 / 18$ & $6.1(4.4-46.5)$ & 0.99 & Ref. & \\
\hline 2nd tertile $(>2.4-4.9)$ & $17 / 17$ & $10.1(6.9-18.3)$ & & $1.05(0.50-2.19)$ & 0.89 \\
\hline 3rd tertile $(>4.9)$ & $17 / 18$ & $9.3(5.7-18.5)$ & & $1.03(0.49-2.14)$ & 0.93 \\
\hline De novo metastatic & $15 / 15$ & $9.8(6.8-15.7)$ & & $1.12(0.53-2.36)$ & 0.77 \\
\hline Linear & & & & $0.98(0.92-1.02)$ & 0.25 \\
\hline \multicolumn{6}{|c|}{ Previous chemotherapy lines, $n$} \\
\hline$\leq 2$ & $30 / 32$ & $10.1(6.9-16.4)$ & 0.11 & Ref. & \\
\hline$>2$ & $34 / 36$ & $8.5(5.5-10.7)$ & & $1.53(0.99-2.59)$ & 0.11 \\
\hline Linear & & & & $1.09(0.90-1.31)$ & 0.38 \\
\hline
\end{tabular}


Table 4 (continued)

\begin{tabular}{|c|c|c|c|c|c|}
\hline & $\begin{array}{l}\text { Number of } \\
\text { events/patients }\end{array}$ & $\begin{array}{l}\text { Median OS, months } \\
(95 \% \mathrm{CI})\end{array}$ & Log rank $p$-value & Hazard Ratio (95\% CI) & Wald $p$-value \\
\hline \multicolumn{6}{|l|}{ ADPT, months } \\
\hline 1st tertile $(\leq 7.8)$ & $21 / 23$ & $4.4(3.5-10.2)$ & \multirow[t]{4}{*}{0.16} & Ref. & \\
\hline 2nd tertile $(>7.8-12.4)$ & $21 / 22$ & $9.3(6.9-16.9)$ & & $0.65(0.35-1.20)$ & 0.16 \\
\hline 3rd tertile $(>12.4)$ & $22 / 23$ & $13.7(8.7-16.5)$ & & $0.56(0.31-1.04)$ & 0.064 \\
\hline Linear & & & & $0.97(0.92-1.02)$ & 0.25 \\
\hline \multicolumn{6}{|l|}{ TNBC } \\
\hline \multicolumn{6}{|l|}{ DRFi, years } \\
\hline 1st tertile $(\leq 1.4)$ & $25 / 27$ & $4.4(2.4-6.6)$ & \multirow[t]{5}{*}{0.36} & Ref. & \\
\hline 2nd tertile $(>1.4-2.8)$ & $26 / 27$ & $5.6(3.9-9.6)$ & & $0.83(0.47-1.46)$ & 0.52 \\
\hline 3rd tertile $(>2.8)$ & $25 / 26$ & $8.9(4.9-15.6)$ & & $0.61(0.34-1.07)$ & 0.085 \\
\hline De novo metastatic & $13 / 13$ & $6.4(3.4-*)$ & & $0.72(0.36-1.41)$ & 0.34 \\
\hline Linear & & & & $0.99(0.92-1.06)$ & 0.75 \\
\hline \multicolumn{6}{|c|}{ Previous chemotherapy lines, $\mathrm{n}$} \\
\hline$\leq 2$ & $64 / 67$ & $6.2(4.9-9.2)$ & \multirow[t]{3}{*}{0.72} & Ref. & \\
\hline$>2$ & $25 / 25$ & $4.6(3.9-9.6)$ & & $1.09(0.68-1.75)$ & 0.72 \\
\hline Linear & & & & $1.01(0.82-1.26)$ & 0.89 \\
\hline \multicolumn{6}{|l|}{ ADPT, months } \\
\hline 1st tertile $(\leq 5.0)$ & $31 / 31$ & $4.3(2.5-5.4)$ & \multirow[t]{4}{*}{0.004} & Ref. & \\
\hline 2nd tertile $(>5.0-8.7)$ & $30 / 30$ & $6.3(3.9-9.0)$ & & $0.81(0.48-1.36)$ & 0.43 \\
\hline 3rd tertile $(>8.7)$ & $28 / 30$ & $12.1(6.5-15.9)$ & & $0.42(0.25-0.71)$ & 0.001 \\
\hline Linear & & & & $0.93(0.88-0.97)$ & 0.002 \\
\hline
\end{tabular}

$A D P T$ average duration of previous treatment lines, $D R F i$ distant recurrence-free interval, $P F S$ progression-free survival

than 2 months and an OS of about 4 months. In contrast an ADPT in the upper tertile, of say 10 months, would predict a doubled PFS and an OS of over a year. This approach would be useful to help not only decide whether to accept the offer of further therapy but also for future planning including end of life care.

The study also has limitations. With all real-world studies, it is difficult to account for all possible confounding factors that may influence the results. The annotations in the medical records were not considered sufficiently comprehensive to present data on adverse events, except for hospitalisation. Performance status and comorbidities were relatively poorly documented in comparison to clinical trial annotations. In addition, our time frame of 7 years allowed for variability of available treatment within each biological subtype, which may have affected the outcome of PFS and OS in our cohort.

In conclusion, by introducing the variable ADPT, we show that patients who have a longer exposure to previous treatment lines have better outcomes on eribulin and this was particularly evident for patients with TNBC. This composite measure has clinical utility for patient information and decision-making at a difficult point in their metastatic journey and should be validated in additional cohorts.
Funding Open access funding provided by University of Gothenburg. No funding was required to conduct this study. S.J.H. receives support from the Manchester NIHR Biomedical Research Centre. M.E. conducted a fellowship at The Christie NHS Foundation Trust, which was funded by research grants from The Swedish Society of Medicine, Futurum - the Academy for Health and Care, The Clinical Cancer Research Foundation in Jönköping, The Percy Falk Foundation, and The Swedish Breast Cancer Association.

Data availability Data are available on request to the corresponding author.

\section{Declarations}

Conflict of interest None of the authors have any competing interests, financial and non-financial, in relation to the work described.

Ethical approval Service evaluation in England is exempt from ethics committee review (Health Research Authority Guidance; www.HRA. NHS.UK). The project was approved by The Christie NHS Foundation Trust as a service evaluation (IRB Reference Number 16/1795).

Consent for publication Not applicable.

Open Access This article is licensed under a Creative Commons Attribution 4.0 International License, which permits use, sharing, adaptation, distribution and reproduction in any medium or format, as long as you give appropriate credit to the original author(s) and the source, provide a link to the Creative Commons licence, and indicate 
if changes were made. The images or other third party material in this article are included in the article's Creative Commons licence, unless indicated otherwise in a credit line to the material. If material is not included in the article's Creative Commons licence and your intended use is not permitted by statutory regulation or exceeds the permitted use, you will need to obtain permission directly from the copyright holder. To view a copy of this licence, visit http://creativecommons. org/licenses/by/4.0/.

\section{References}

1. Bray F, Ferlay J, Soerjomataram I, Siegel RL, Torre LA, Jemal A (2018) Global cancer statistics 2018: GLOBOCAN estimates of incidence and mortality worldwide for 36 cancers in 185 countries. Cancer J Clin 68(6):394-424. https://doi.org/10.3322/caac. 21492

2. Cardoso F, Paluch-Shimon S, Senkus E, Curigliano G, Aapro MS, André F, Barrios CH, Bergh J, Bhattacharyya GS, Biganzoli L, Boyle F, Cardoso MJ, Carey LA, Cortés J, El Saghir NS, Elzayat M, Eniu A, Fallowfield L, Francis PA, Gelmon K, Gligorov J, Haidinger R, Harbeck N, Hu X, Kaufman B, Kaur R, Kiely BE, Kim SB, Lin NU, Mertz SA, Neciosup S, Offersen BV, Ohno S, Pagani O, Prat A, Penault-Llorca F, Rugo HS, Sledge GW, Thomssen C, Vorobiof DA, Wiseman T, Xu B, Norton L, Costa A, Winer EP (2020) 5th ESO-ESMO international consensus guidelines for advanced breast cancer (ABC 5). Ann Oncol 31(12):1623-1649. https://doi.org/10.1016/j.annonc.2020.09.010

3. Cortes J, O'Shaughnessy J, Loesch D, Blum JL, Vahdat LT, Petrakova K, Chollet P, Manikas A, Dieras V, Delozier T, Vladimirov V, Cardoso F, Koh H, Bougnoux P, Dutcus CE, Seegobin S, Mir D, Meneses N, Wanders J, Twelves C, investigators E (2011) Eribulin monotherapy versus treatment of physician's choice in patients with metastatic breast cancer (EMBRACE): a phase 3 open-label randomised study. Lancet 377(9769):914-923. https:// doi.org/10.1016/S0140-6736(11)60070-6

4. Kaufman PA, Awada A, Twelves C, Yelle L, Perez EA, Velikova G, Olivo MS, He Y, Dutcus CE, Cortes J (2015) Phase III openlabel randomized study of eribulin mesylate versus capecitabine in patients with locally advanced or metastatic breast cancer previously treated with an anthracycline and a taxane. J Clin Oncol 33(6):594-601. https://doi.org/10.1200/jco.2013.52.4892

5. Pivot X, Im SA, Guo M, Marmé F (2018) Subgroup analysis of patients with HER2-negative metastatic breast cancer in the second-line setting from a phase 3, open-label, randomized study of eribulin mesilate versus capecitabine. Breast Cancer 25(3):370374. https://doi.org/10.1007/s12282-017-0826-4

6. Twelves C, Cortes J, Vahdat L, Olivo M, He Y, Kaufman PA, Awada A (2014) Efficacy of eribulin in women with metastatic breast cancer: a pooled analysis of two phase 3 studies. Breast Cancer Res Treat 148(3):553-561. https://doi.org/10.1007/ s10549-014-3144-y

7. Inoue K, Takahashi M, Mukai H, Yamanaka T, Egawa C, Sakata Y, Ikezawa H, Matsuoka T, Tsurutani J (2020) Effectiveness and safety of eribulin in Japanese patients with HER2-negative, advanced breast cancer: a 2-year post-marketing observational study in a real-world setting. Invest New Drugs 38(5):1540-1549. https://doi.org/10.1007/s10637-019-00890-5
8. Gamucci T, Michelotti A, Pizzuti L, Mentuccia L, Landucci E, Sperduti I, Di Lauro L, Fabi A, Tonini G, Sini V, Salesi N, Ferrarini I, Vaccaro A, Pavese I, Veltri E, Moscetti L, Marchetti P, Vici P (2014) Eribulin mesylate in pretreated breast cancer patients: a multicenter retrospective observational study. J Cancer 5(5):320-327. https://doi.org/10.7150/jca.8748

9. Zhao Y, Xie N, Li W, Chen W, Lv Z, Zheng Y, Sun T, Liu J, Zhang J, Hu S, Wang Y, Gong C, Li Y, Xie Y, Ge R, Xu F, Wang B (2021) Real-world effectiveness of eribulin in heavily pretreated patients with metastatic breast cancer in China: a multicenter retrospective study. Ther Adv Med Oncol 13:17588359211030210. https://doi.org/10.1177/17588359211030210

10. Mougalian SS, Kish JK, Zhang J, Liassou D, Feinberg BA (2021) Effectiveness of eribulin in metastatic breast cancer: 10 years of real-world clinical experience in the United States. Adv Ther 38(5):2213-2225. https://doi.org/10.1007/s12325-020-01613-6

11. Park MH, Lee SJ, Noh WC, Jeon CW, Lee SW, Son GS, Moon BI, Lee JS, Kang SS, Suh YJ, Gwak G, Kim TH, Yoo YB, Kim HA, Kim MY, Kim JY, Jeong J (2020) A nationwide, multicenter retrospective study on the effectiveness and safety of eribulin in Korean breast cancer patients (REMARK). Breast 54:121-126. https://doi.org/10.1016/j.breast.2020.09.004

12. Jacot W, Heudel PE, Fraisse J, Gourgou S, Guiu S, Dalenc F, Pistilli B, Campone M, Levy C, Debled M, Leheurteur M, Chaix M, Lefeuvre C, Goncalves A, Uwer L, Ferrero JM, Eymard JC, Petit T, Mouret-Reynier MA, Courtinard C, Cottu P, Robain M, Mailliez A (2019) Real-life activity of eribulin mesylate among metastatic breast cancer patients in the multicenter national observational ESME program. Int J Cancer 145(12):3359-3369. https:// doi.org/10.1002/ijc.32402

13. Adamo V, Ricciardi GRR, Giuffrida D, Scandurra G, Russo A, Blasi L, Spadaro P, Iacono C, Soto Parra HJ, Savarino A, Ferraú F, Zerilli F, Verderame F, Butera A, Santangelo C, Franchina V, Caruso M (2019) Eribulin mesylate use as third-line therapy in patients with metastatic breast cancer (VESPRY): a prospective, multicentre, observational study. Ther Adv Med Oncol 11:1758835919895755. https://doi.org/10.1177/1758835919 895755

14. Garrone O, Montemurro F, Saggia C, La Verde N, Vandone AM, Airoldi M, De Conciliis E, Donadio M, Lucio F, Polimeni MA, Oletti MV, Giacobino A, Merlano MC (2016) Eribulin in pretreated metastatic breast cancer patients: results of the TROTTER trial-a multicenter retrospective study of eribulin in real life. Springerplus 5:59. https://doi.org/10.1186/s40064-016-1700-0

15. Swain SM, Miles D, Kim SB, Im YH, Im SA, Semiglazov V, Ciruelos E, Schneeweiss A, Loi S, Monturus E, Clark E, Knott A, Restuccia E, Benyunes MC, Cortés J (2020) Pertuzumab, trastuzumab, and docetaxel for HER2-positive metastatic breast cancer (CLEOPATRA): end-of-study results from a double-blind, randomised, placebo-controlled, phase 3 study. Lancet Oncol 21(4):519-530. https://doi.org/10.1016/s1470-2045(19)30863-0

16. Cortes J, Twelves C (2020) Impact of the number of prior chemotherapy regimens on outcomes for patients with metastatic breast cancer treated with eribulin: A post hoc pooled analysis. Breast $\mathbf{J}$ 26(7):1347-1351. https://doi.org/10.1111/tbj.13686

Publisher's Note Springer Nature remains neutral with regard to jurisdictional claims in published maps and institutional affiliations. 